\title{
GABAergic Neurons Are Less Selective to Stimulus Orientation than Excitatory Neurons in Layer II/III of Visual Cortex, as Revealed by In Vivo Functional $\mathrm{Ca}^{2+}$ Imaging in Transgenic Mice
}

\author{
Kazuhiro Sohya ${ }^{1,2 \star}$ Katsuro Kameyama, ${ }^{1,2 \star}$ Yuchio Yanagawa, ${ }^{2,3}$ Kunihiko Obata, ${ }^{1}$ and Tadaharu Tsumoto ${ }^{1,2}$ \\ ${ }^{1}$ Brain Science Institute, RIKEN, Wako 351-0198, Japan, ${ }^{2}$ Solution-Oriented Research for Science and Technology, Japan Science and Technology Agency, \\ Kawaguchi 442-0012, Japan, and ${ }^{3}$ Gunma University Graduate School of Medicine, Maebashi 371-8511, Japan
}

\begin{abstract}
Most neurons in the visual cortex are selectively responsive to visual stimulation of a narrow range of orientations, and GABAergic neurons are considered to play a role in the formation of such orientation selectivity. This suggests that response properties of GABAergic neurons may be different from those of excitatory neurons. This view remains unproved, however. To address this issue, we applied in vivo two-photon functional $\mathrm{Ca}^{2+}$ imaging to transgenic mice, in which GABAergic neurons express enhanced green fluorescent protein. Astroglia were stained by an astrocyte-specific dye. The three types of cells, GABAergic neurons, excitatory neurons, and astrocytes, in layer II/III of the visual cortex were differentially identified by using different wavelengths of excitation light and a dichroic mirror for emitted fluorescence, and their responses to moving visual stimuli at different orientations were measured with changes in the intensity of fluorescence of $\mathrm{C}^{2+}$-sensitive dye. We found that almost all GABAergic neurons have orientation-insensitive responses, whereas most of excitatory neurons have orientation-selective responses.
\end{abstract}

Key words: GABAergic neurons; orientation selectivity; visual cortex; two-photon functional calcium imaging; transgenic mice; green fluorescent protein

\section{Introduction}

The cerebral cortex consists mainly of two types of neurons, glutamatergic excitatory and GABAergic inhibitory neurons. One of the principal functions of GABAergic neurons is considered to be the sharpening of specific functional properties of cortical neurons. In the visual cortex, for example, GABAergic neurons were proposed to play a role in rendering cortical neurons selective to a particular orientation of visual stimuli, although this view still remains highly controversial (Sillito, 1975; Tsumoto et al., 1979; Ferster, 1986; Chapman et al., 1991; Somers et al., 1995; Shevelev et al., 1998; Anderson et al., 2000; Teich and Qian, 2006). If GABAergic neurons play such a role, they are assumed to have the preferred orientation orthogonal to or different from that of neighboring excitatory neurons to curb nonpreferred inputs or to have no or poor selectivity to generally suppress orientationbiased inputs to excitatory neurons (for review, see Vidyasagar et al., 1996; Sompolinsky and Shapley, 1997; Ferster and Miller,

\footnotetext{
Received 0ct. 25, 2006; revised Jan. 24, 2007; accepted Jan. 26, 2007.

We express our deepest gratitude to Drs. K. Ohki, T. Nemoto, M. Matsuzaki, and N. Takata for technical advice on in vivo two-photon functional $\mathrm{Ca}^{2+}$ imaging. We also thank Drs. H. Hirase and B. Jiang for critically reading this manuscript.

*K.S. and K.K. contributed equally to this work.

Correspondence should be addressed to Dr. Tadaharu Tsumoto, Brain Research Institute, RIKEN, 2-1 Hirosawa, Wako 351-0198, Japan. E-mail: tsumoto@brain.riken.jp.

D0I:10.1523/JNEUROSCI.4641-06.2007

Copyright $\odot 2007$ Society for Neuroscience $\quad$ 0270-6474/07/272145-05\$15.00/0
}

2000). To date, however, the possible differences in response properties of the two types of cortical neuron have not directly been tested by simultaneous recordings of their visual responses in the visual cortex in vivo, although visual responses of "suspected interneurons" in the rabbit visual cortex and "smooth" interneurons in layer IV of the cat visual cortex were reported in previous studies (Swadlow, 1988; Hirsch et al., 2003). By application of in vivo two-photon functional $\mathrm{Ca}^{2+}$ imaging to transgenic mice, we found that almost all GABAergic neurons, which were identified by enhanced green fluorescent protein (EGFP), have orientation-insensitive responses, whereas most excitatory neurons have orientation-selective responses.

\section{Materials and Methods}

Animal preparations. GAD67-GFP ( $\Delta$ neo) mice (Tamamaki et al., 2003) at postnatal weeks $8-12$ were anesthetized with urethane $(1.9 \mathrm{mg} / \mathrm{g}$ body weight), supplemented with additional doses $(0.25 \mathrm{mg} / \mathrm{g})$ of urethane when necessary. Incisions were infiltrated with lidocaine (xylocaine jelly). The animal's body temperature was maintained at $37.5^{\circ} \mathrm{C}$ by a rectal thermoprobe feeding back to a heating pad (NS-TC10; Neuroscience, Tokyo, Japan). All experimental procedures were performed in accordance with the guidelines of the Animal Experiments Committee of RIKEN Brain Science Institute. The part of the skull and dura mater over the primary visual cortex was removed, and the exposed cortex was covered with agarose (2-3\% in Ringer's solution). Qualities of these transgenic mice and additional descriptions about the methods are detailed in the supplemental Materials and Methods (available at www.jneurosci. org as supplemental material). 
Multicell bolus dye loading and in vivo two-photon microscopy. A total of $10 \mathrm{~mm}$ fura-2 AM (Invitrogen, Eugene, OR) was dissolved in dimethylsulfoxide mixed with $20 \%$ Pluronic F-127 (Invitrogen) and then diluted 10 times with $0.1 \mathrm{~mm}$ sulforhodamine 101 (SR101; Invitrogen) that was dissolved in Ringer's solution. A glass pipette with a tip diameter of $\sim 10$ $\mu \mathrm{m}$ was filled with this solution and inserted into the cortex to a depth of $150-200 \mu \mathrm{m}$ from the surface. Fura-2 AM and SR101 were ejected from the pipettes by pressure. Images of cells stained by the dyes were visualized using a two-photon laser-scanning system (Radiance 2000MP; BioRad, Hertfordshire, UK) coupled with a mode-locked Ti:sapphire laser (pulse width of $<100 \mathrm{fs}, 80 \mathrm{MHz}$ repetition frequency at a wavelength of $800 \mathrm{~nm}$; Tsunami; Spectra-Physics, Mountain View, CA) pumped with a $10 \mathrm{~W}$ solid-state source (Millenia PRO 10sJ 110; Spectra-Physics). The scanhead was attached to an upright microscope (ECLIPSE E600FN; Nikon, Tokyo, Japan). Excitation light was focused using a $40 \times$ waterimmersion objective ( 0.8 numerical aperture; Nikon). Emitted fluorescence was divided into long- (>570 nm) and short-wavelength light with a dichroic mirror (570 nm; Olympus, Tokyo, Japan), and shortwavelength light was further filtered through a bandpass filter (510-550 $\mathrm{nm}$; Olympus) as an emission filter. Both wavelengths of emitted fluorescence were detected simultaneously using two photomultiplier tube detectors (PMTs; Direct Detection System; Bio-Rad). The PMT offset was kept at zero throughout the recordings. The microscope objective was shielded from possible stray light by covering the space over the animal's head with lightproof cloth.

Data acquisition and analysis. A square region of the cortex 150-300 $\mu \mathrm{m}$ on one side was imaged at $512 \times 512$ pixels, skipping every other line at $0.34-0.36 \mathrm{~s}$ per frame. In all experiments, images were obtained from at least three depths separated by $10-15 \mu \mathrm{m}$ in most cases and $5 \mu \mathrm{m}$ in exceptional cases. Time-lapse images were realigned to remove tangential drifts, and cell regions in the images were contoured visually with custom-made software. In some experiments, the images were realigned using MetaMorph software (Molecular Devices, Downingtown, PA). A fura-2 signal was detected as a decrease in fluorescence intensity, which was calculated as $-\Delta F / F_{0}$, in which $\Delta F=F-F_{0}$. The baseline intensity, $F_{0}$, was obtained by averaging the intensity values during the prestimulus period ( $5 \mathrm{~s}$ ). The baseline signals of cells were computed by summing pixel values within the defined cell contours.

Visual stimulation and responses. Square wave gratings were moved on an LCD monitor in eight directions (from 0 to $315^{\circ}$ in $45^{\circ}$ steps). These eight patterns of visual stimuli were presented usually five times in a randomly interleaved manner, and then signals for $25 \mathrm{~s}$ ( $5 \mathrm{~s}$ before, $5 \mathrm{~s}$ during, and $15 \mathrm{~s}$ after the presentation of each angle of stimuli) were rearranged in the order of angle so that signals for the same angle of stimuli were averaged. To obtain the response magnitude $(R \theta)$ at each of the eight angles, intensity values during the presentation of the stimuli at each angle were averaged. In this averaging, negative values were regarded as zero, because they were not often observed in visually responsive cells and, even if they existed, they were randomly dispersed. Cells were defined as visually responsive if the largest one of the eight $R \theta \mathrm{s}$ was larger than 3 SDs of the baseline intensity during prestimulation periods. The orientation selectivity index (OSI) of visual responses was calculated as follows:

$$
\text { OSI }=\sqrt{\left(\Sigma R\left(\theta_{i}\right) \sin \left(2 \theta_{i}\right)\right)^{2}+\left(\Sigma R\left(\theta_{i}\right) \cos \left(2 \theta_{i}\right)\right)^{2}} / \Sigma R\left(\theta_{i}\right)
$$

where $\theta$ is the angle of the directed grating stimuli ranging from 0 to $315^{\circ}$, and $R(\theta)$ is the response magnitude at each angle. $R(\theta)$ was also used as the point of polar diagrams.

\section{Results}

\section{Separate visualization of three groups of cells}

To analyze the responses of neurons to visual stimuli, we injected fura-2 AM, a Ca ${ }^{2+}$-sensitive indicator (Grynkiewicz et al., 1985), together with SR101, which specifically stains astrocytes (Nimmerjahn et al., 2004), into layer II/III of the visual cortex of GAD67-GFP $(\Delta$ neo $)$ mice through glass micropipettes. For two-

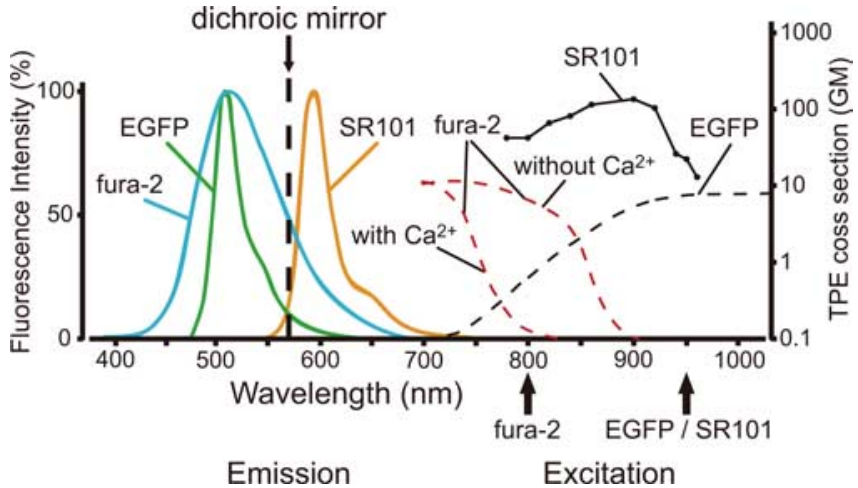

Figure 1. Spectra of emission light (left) and two-photon excitation (TPE) cross section (right) for the three fluorescent dyes. Three curves in the left half schematically show the intensity of fluorescence emitted from each of the dyes as values normalized to the maximum, modified from Fluorescence Spectra Viewer (Invitrogen). The ordinates on the left and right sides apply to the three curves in the left half and TPE cross-section spectra curves in the right half, respectively. Two dashed red curves schematically show TPE cross-section spectra of fura-2 bound and not bound with $\mathrm{Ca}^{2+}$, modified from Xu et al. (1996). The dotted curve for EGFP schematically shows its TPE cross-section spectra, modified also from Xu et al. (1996). The solid curve for SR101 schematically shows its TPE cross-section spectra measured at 780-960 nm excitation wavelength in aqueous solution. TPE cross-section values of Goeppert-Mayer unit (GM) of SR101 were obtained by comparing the measured intensity of fluorescence with that of Rhodamine B, on the following assumptions: the two-photon absorption cross section of Rhodamine $B$ in aqueous solution is the same as that in methanol solution (210 GM at $840 \mathrm{~nm}$ two-photon excitation); the two-photon fluorescence quantum efficiency of Rhodamine $B$ is the same as its one-photon fluorescence quantum efficiency (0.7); and the sample refractive index in aqueous solution is the same as that in methanol solution (Albota et al., 1998).

photon excitation of fura-2, we used laser light at a wavelength of $800 \mathrm{~nm}(790-810 \mathrm{~nm}$ at half-height), which does not effectively excite EGFP (Fig. 1). Usually, the laser power for fura-2 excitation was set at $10 \mathrm{~mW}$ to minimize possible bleaching of the dye and toxicity to cells. When fura- 2 is bound with $\mathrm{Ca}^{2+}$, excitation efficacy (two-photon excitation cross section) decreased so that an increase in the concentration of $\mathrm{Ca}^{2+}$ is expressed as a decrease in the intensity of emitted fluorescence (Fig. 1, dashed red curves).

After the measurement of $\mathrm{Ca}^{2+}$ signal, EGFP-positive cells (GABAergic neurons) and SR101-positive cells (astrocytes) were identified in the cortex in vivo, using laser light at an excitation wavelength of $950 \mathrm{~nm}$ (940-960 nm at half-height), which does not excite fura-2 (Fig. 1). Although the excitation wavelength was the same, emitted fluorescent wavelengths of EGFP and SR101 were completely different, and thus we could separate their fluorescence signals with a dichroic mirror at $570 \mathrm{~nm}$ (Fig. 1, dashed vertical line). By superimposing the images obtained as described above, we could identify the three groups of cells, EGFP- and SR101-negative cells (excitatory neurons), EGFP-positive and SR101-negative cells (GABAergic neurons), and EGFP-negative and SR101-positive cells (astrocytes). EGFP- and SR101-positive cells were not observed.

Such a separate visualization of the three groups of cells was confirmed in another series of in vivo experiments (Fig. 2). The visual cortex of wild-type mice was loaded with fura-2 and SR101. At the excitation wavelength of $800 \mathrm{~nm}$, signals of fura- 2 were visible in a channel for emission light $<570 \mathrm{~nm}$ (Fig. $2 \mathrm{Aa}$ ). When the excitation wavelength was changed to $950 \mathrm{~nm}$ with the laser power of $\sim 70 \mathrm{~mW}$, almost none of the cells were detected in a channel for emission light $<570 \mathrm{~nm}$ (Fig. 2Ab), but SR101positive cells were clearly visible in the other channel for emission light $>570 \mathrm{~nm}$ (Fig. 2Ac). In the visual cortex of GAD67-GFP 


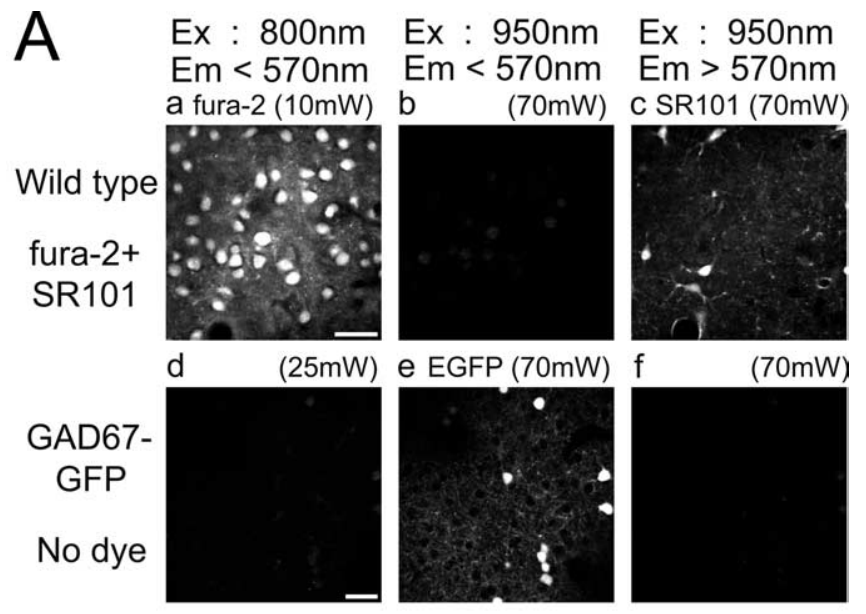

B a Ex : $800 \mathrm{~nm}$ $\mathrm{Em}<570 \mathrm{~nm}$
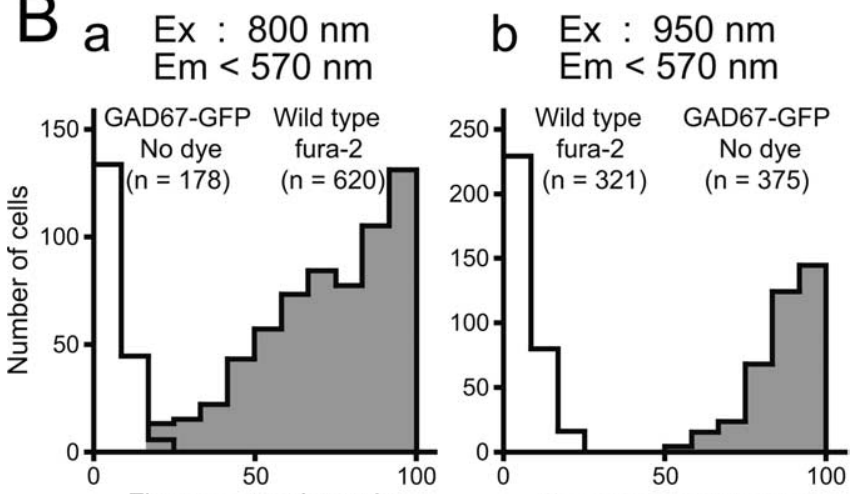

Fluorescence Intensity

Fluorescence Intensity

Figure 2. Separate visualization of three groups of cells. $A$, Visibility or invisibility of cortical cells of wild-type (top) and GAD67-GFP ( $\Delta$ neo; bottom) mice under the conditions indicated at the top. The cortex of the former mice was loaded with fura-2 and SR101, whereas that of the latter was not loaded with any dye. Scale bars in the images in the left column indicate $30 \mu \mathrm{m}$ and apply to the images in the same row. The plane of the depth was $155 \mu \mathrm{m}$ in $\boldsymbol{a}$-c and 150 $\mu \mathrm{m}$ in $\boldsymbol{d}-\boldsymbol{f}$. $\boldsymbol{B}$, Distributions of the emitted fluorescence intensity of EGFP-expressing neurons in the visual cortex of GAD67-GFP $(\Delta$ neo $)$ mice and that of fura-2-loaded neurons in the visual cortex of wild-type mice, at excitation wavelengths of $800 \mathrm{~nm}(\boldsymbol{a})$ and $950 \mathrm{~nm}(\boldsymbol{b})$. Fluorescence intensity was normalized to the maximum value. The PMT gain was constant in the same excitation and emission conditions.

$(\Delta$ neo $)$ mice that were not loaded with any dye but expressed EGFP in GABAergic neurons, almost no cells were visualized at the excitation wavelength of $800 \mathrm{~nm}$ even if the laser power was increased to $25 \mathrm{~mW}$ (Fig. $2 \mathrm{Ad}$ ). At the excitation wavelength of $950 \mathrm{~nm}$, EGFP-positive cells were visible in a channel for emission light $<570 \mathrm{~nm}$ (Fig. $2 \mathrm{Ae}$ ) but not detected in the other channel for emission light $>570 \mathrm{~nm}(A f)$.

To quantitatively confirm that $\mathrm{Ca}^{2+}$ signals of fura- 2 in EGFP-positive neurons were not contaminated with EGFP signals, the fluorescence intensity of 620 cells in the fura-2-loaded cortex of wild-type mice was measured and compared with that of 178 EGFP-positive cells in the visual cortex of GAD67-GFP $(\Delta$ neo) mice under the conditions for fura-2 signal measurements (Fig. $2 \mathrm{Ba}$ ). The fluorescence intensity of almost all of the cells in the GAD67-GFP ( $\Delta$ neo) mice was nearly zero, and there was almost no overlap of the fluorescence intensity with that of the fura-2-loaded neurons. To further confirm that $\mathrm{Ca}^{2+}$ signals of fura- 2 were not contaminated with EGFP signals, the intensity of fluorescence was measured from 321 fura-2-loaded cells in the visual cortex of wild-type mice under the conditions for excitation of EGFP (Fig. 2 Bb). Almost none of the 321 fura-2-loaded cells showed detectable fluorescence under the conditions in which most of the 375 EGFP-positive cells in GAD67-GFP $(\Delta$ neo) mice showed robust fluorescence.

The results shown in Figures 1 and 2 indicate that $\mathrm{Ca}^{2+}$ signals of fura- 2 were not contaminated with EGFP signals.

\section{Difference in orientation tuning between GABAergic and non-GABAergic neurons}

In the in vivo visual cortex of GAD67-GFP ( $\Delta$ neo) mice that were loaded with fura-2 and SR101, fura-2-loaded cells were visible at the excitation wavelength of $800 \mathrm{~nm}$ in a channel for emission light $<570 \mathrm{~nm}$ (Fig. 3A-C). At the excitation wavelength of 950 $\mathrm{nm}$, EGFP-positive and SR101-positive cells were differentially visualized in channels for emission light lower and higher than $570 \mathrm{~nm}$, respectively. In Figure $3 A-C$, they are shown as green and red cells, respectively. As seen in these figures, EGFP-positive cells were observed to be scattered in the cortex. It is established that almost all of the EGFP-positive cells are GABAergic (Tamamaki et al., 2003). SR101-positive cells were distributed sparsely in the present preparations.

Changes in $\mathrm{Ca}^{2+}$ signals of cells were assessed by summing pixel values of fura-2 fluorescence within contours of somata. As established previously (Mao et al., 2001; Stosiek et al., 2003; Kerr et al., 2005), somatic $\mathrm{Ca}^{2+}$ signals reflect action potentials rather than subthreshold depolarization. Responses of fura-2-labeled cells to visual stimuli were studied using gratings at four different orientations, each drifting in two opposite directions. The response magnitude to each of the eight stimulus patterns was obtained from the averaged value during the stimulus presentation. When fluorescence signals were judged as mixed with strong spontaneous activities or with artifacts attributable to movements of the cortex, the data were excluded from the analysis. In addition to measurement of changes in fluorescence in cell bodies, changes in adjacent neuropils were also observed (Fig. 3C).

We analyzed changes in fluorescence intensity of 664 cells from 13 focal planes of the visual cortex in four transgenic mice. If the same cells were observed in different planes, only one of the images that showed larger responses was analyzed. Cells that were not clearly separated from neighboring cells were excluded from the analysis. We found that 181 of the 533 GFP- and SR101negative neurons that were judged as excitatory neurons showed significant responses, and most of the 181 neurons responded preferentially to a particular orientation of stimuli. Among the 70 GFP-positive and SR101-negative cells that were judged as GABAergic neurons, 28 showed visual responses, and most of these 28 neurons responded nonpreferentially to different orientations of stimuli. Only one of the 61 SR101-positive and GFPnegative cells that were judged as astrocytes had detectable responses to visual stimuli.

Examples of visual responses are shown in Figure 3D. Excitatory neurons designated as cells 1 and 2 in Figure $3 C$ responded best to the motion of horizontally oriented stimuli (Fig. $3 D$, the first and second sweeps). However, these cells did not show such robust responses to motion of vertically and obliquely oriented stimuli. Polar plots of the response magnitudes confirmed a sharp tuning of the responses (Fig. 3E). In contrast, a GABAergic neuron (cell 3) showed robust responses to all of the orientations of stimuli (Fig. 3D, third sweep). A polar plot confirmed orientation-insensitive responses (Fig. $3 E$, right). We also observed that all of the nearby areas containing neuropils $(a-c)$ showed orientation-insensitive responses with the similar magnitude and pattern, although the magnitude was much smaller than that of cell 3. The orientation-selective responses of the cells 
1 and 2 and the much larger responses of cell 3 than those of neuropils confirmed that neuropil signals did not significantly disturb the signal of neuronal responses, and thus the two-photon $\mathrm{Ca}^{2+}$ imaging in the present preparations has single-cell resolution (Stosiek et al., 2003; Ohki et al., 2005). Additional examples of responses of GFP-negative and -positive neurons are shown in supplemental Figure 1 (available at www.jneurosci.org as supplemental material).

We calculated the OSI of all of the cells with significant visual responses using the vector averaging method (Swindale, 1998). The distributions of OSIs of all of the excitatory and GABAergic neurons that were visually responsive are shown in Figure 4. As shown in Figure 4A, the OSIs of excitatory neurons ranged from 0.01 (nonoriented) to 0.86 (orientation selective). The mean value was $0.32 \pm 0.16$ (SD). In contrast, the distribution of OSIs of GABAergic neurons was strongly skewed toward zero. The range and mean were $0.02-0.42$ and $0.13 \pm 0.08$, respectively. The distributions of OSIs of both types of neuron were significantly different (Kolmogorov-Smirnov test, $p<$ 0.001 ). We also found that the peak fluorescence intensity of visual responses of GABAergic neurons was not significantly different, but the rising slope was smaller than that of excitatory neurons (supplemental Fig. 2, available at www.jneurosci. org as supplemental material).

The present results demonstrate that almost all of the inhibitory neurons in the visual cortex showed orientation-insensitive responses, whereas most of the excitatory neurons showed orientation-selective responses.

\section{Discussion}

In the present study, 181 (34\%) of the 533 non-GABAergic neurons and $28(40 \%)$ of the 70 GABAergic neurons had significant visual responses. These proportions of visually responsive neurons are similar to that (37\%) of the rat visual cortex reported by Ohki et al. (2005), although they did not classify the neurons into non-GABAergic and GABAergic. Previous studies using microelectrodes in mouse visual cortex reported that $\sim 90 \%$ of neurons were visually responsive (Draeger, 1975; Mangini and Pearlman, 1980; Metin et al., 1988). As discussed by Ohki et al. (2005), a factor for the relatively small proportion of visually responsive neurons in the in vivo functional $\mathrm{Ca}^{2+}$ imaging study may be ascribable to the application of visual stimulation with the single parameters (e.g., frequency and contrast of gratings and velocity of movements) except for orientation and movement direction to a population of neurons that are supposed to have different optimal responses to various parameters of visual stimuli. There is another possibility that anesthesia might reduce visual responsiveness (see supplemental Discussion, available at www. jneurosci.org as supplemental material).

GABAergic neurons are classified into various types, such as basket cells, bipolar cells, etc. on the basis of morphology; fast-
A

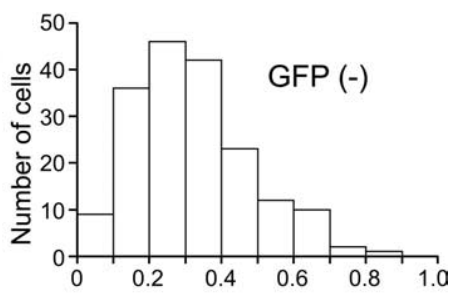

B

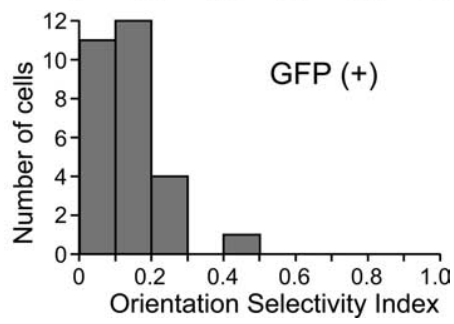

Figure 4. $A, B$, Distribution of OSI of visual responses of GFP-negative and -positive neurons, respectively. The total numbers of cells in $\boldsymbol{A}$ and $\boldsymbol{B}$ are 181 and 28 , respectively.

spiking cells, burst-spiking cells, etc. on the basis of physiological properties; and parvalbumin-positive cells, somatostatinpositive cells, etc. on the basis of neurochemical properties (Kawaguchi and Kubota, 1997). In the present study, we did not classify EGFP-positive neurons into subgroups of GABAergic neurons. Therefore, we cannot exclude the possibility that the visually responsive EGFP-positive neurons in the present study might be biased to a particular group of GABAergic neurons. 
We observed that almost all of the areas representing neuropils in which measurements were performed showed orientationinsensitive responses with a relatively small magnitude. This seems consistent with a previous report suggesting that optical $\mathrm{Ca}^{2+}$ signals from neuropils mostly originate from axonal structures, presumably reflecting the bulk average of action-potentialevoked $\mathrm{Ca}^{2+}$ transients in presynaptic structures (Kerr et al., 2005). In the present study, recordings were performed from layer II/III of the visual cortex. In the primary visual cortex of the mouse, afferent axons in layer II/III originate mostly from cortical layer IV neurons and to some degree from relay neurons of the dorsal lateral geniculate nucleus (dLGN) (Frost and Caviness, 1980). Most layer IV neurons of the mouse visual cortex have nonoriented receptive fields, and also, dLGN relay neurons have nonoriented visual responses (Draeger, 1975; Mangini and Pearlman, 1980; Grubb and Thompson, 2003).

As mentioned in the Introduction, the questions of whether GABAergic inhibition plays a role in the formation of orientation selectivity of visual cortex neurons, and if so, what role they play, are still highly controversial. The present results seem consistent with the view that nonoriented or broadly tuned cortical inhibition raises the firing threshold of excitatory neurons so as to make their responses more sharply tuned.

The relatively slow rising slope of optimal visual responses of GABAergic neurons, a possibility of saturation of the responses, and sources of their nonoriented responses are discussed in supplemental Discussion (available at www.jneurosci.org as supplemental material).

\section{References}

Albota MA, Xu C, Webb WW (1998) Two-photon fluorescence excitation cross sections of biomolecular probes from 690 to $960 \mathrm{~nm}$. Appl Opt 37:7352-7356.

Anderson JS, Carandini M, Ferster D (2000) Orientation tuning of input conductance, excitation, and inhibition in cat primary visual cortex. J Neurophysiol 84:909-926.

Chapman B, Zahs KR, Stryker MP (1991) Relation of cortical cell orientation selectivity to alignment of receptive fields of the geniculocortical afferents that arborize within a single orientation column in ferret visual cortex. J Neurosci 11:1347-1358.

Draeger UC (1975) Receptive fields of single cells and topography in mouse visual cortex. J Comp Neurol 160:269-287.

Ferster D (1986) Orientation selectivity of synaptic potentials in neurons of cat primary visual cortex. J Neurosci 6:1284-1301.

Ferster D, Miller KD (2000) Comparison among some models of orientation selectivity. Annu Rev Neurosci 23:441-471.

Frost DO, Caviness Jr VS (1980) Radial organization of thalamic projections to the neocortex in the mouse. J Comp Neurol 194:369-393.

Grubb MS, Thompson ID (2003) Quantitative characterization of visual response properties in the mouse dorsal lateral geniculate nucleus. J Neurophysiol 90:3594-3607.

Grynkiewicz G, Poenie M, Tsien RY (1985) A new generation of $\mathrm{Ca}^{2+}$ indi- cators with greatly improved fluorescence properties. J Biol Chem 260:3440-3450.

Hirsch JA, Martinez LM, Pillai C, Alonso J-M, Wang Q, Sommer FT (2003) Functionally distinct inhibitory neurons at the first stage of visual cortical processing. Nat Neurosci 6:1300-1308.

Kawaguchi Y, Kubota Y (1997) GABAergic cell subtypes and their synaptic connections in rat frontal cortex. Cereb Cortex 7:476-486.

Kerr JND, Greenberg D, Helmchen F (2005) Imaging input and output of neocortical networks in vivo. Proc Natl Acad Sci USA 102:14063-14068.

Mangini NJ, Pearlman AL (1980) Laminar distribution of receptive field properties in the primary visual cortex of the mouse. J Comp Neurol 193:203-222.

Mao BQ, Hamzei-Sichani F, Aronov D, Froemke RC, Yuste R (2001) Dynamics of spontaneous activity in neocortical slices. Neuron 32:883-898.

Metin C, Godement P, Imbert M (1988) The primary visual cortex in the mouse: receptive field properties and functional organization. Exp Brain Res 69:594-612.

Nimmerjahn A, Kirchhoff F, Kerr JND, Helmchen F (2004) Sulforhodamine 101 as a specific marker of astroglia in the neocortex in vivo. Nat Methods 1:31-37.

Ohki K, Chung S, Ch'ng YH, Kara P, Reid RC (2005) Functional imaging with cellular resolution reveals precise micro-architecture in visual cortex. Nature 433:597-603.

Shevelev IA, Eysel UT, Lazareva NA, Sharaev GA (1998) The contribution of intracortical inhibition to dynamics of orientation tuning in cat striate cortex neurons. Neuroscience 84:11-23.

Sillito AM (1975) The contribution of inhibitory mechanisms to the receptive field properties of neurones in the striate cortex of the cat. J Physiol (Lond) 250:305-329.

Somers DC, Nelson SB, Sur M (1995) An emergent model of orientation selectivity in cat visual cortical simple cells. J Neurosci 15:5448-5465.

Sompolinsky H, Shapley R (1997) New perspectives on the mechanisms for orientation selectivity. Curr Opin Neurobiol 7:514-522.

Stosiek K, Garachuk O, Holthoff K, Konnerth A (2003) In vivo two-photon calcium imaging of neuronal networks. Proc Natl Acad Sci USA 100:7319-7324.

Swadlow HA (1988) Efferent neurons and suspected interneurons in binocular visual cortex of the awake rabbit: receptive fields and binocular properties. J Neurophysiol 59:1162-1187.

Swindale NV (1998) Orientation tuning curves: empirical description and estimation of parameters. Biol Cybern 78:45-56.

Tamamaki N, Yanagawa Y, Tomioka R, Miyazaki J, Obata K, Kaneko T (2003) Green fluorescence protein expression and colocalization with calretinin, parvalbumin, and somatostatin in the GAD67-GFP knock-in mouse. J Comp Neurol 467:60-79.

Teich AF, Qian N (2006) Comparison among models of orientation selectivity. J Neurophysiol 96:404-419.

Tsumoto T, Eckart W, Creutzfeldt OD (1979) Modification of orientation sensitivity of cat visual cortex neurones by removal of GABA-mediated inhibition. Exp Brain Res 34:351-363.

Vidyasagar TR, Pei X, Volgushev M (1996) Multiple mechanisms underlying the orientation selectivity of visual cortical neurons. Trends Neurosci 19:272-277.

Xu C, Williams RM, Zipfel W, Webb WW (1996) Multiphoton excitation cross-sections of molecular fluorophores. Bioimaging 4:198-207. 